\title{
CONTINUOUS COHOMOLOGY FOR COMPACTLY SUPPORTED VECTORFIELDS ON $\mathrm{R}^{n}$
}

\author{
BY \\ STEVEN SHNIDER( $\left.{ }^{1}\right)$
}

\begin{abstract}
In this paper we study the Gelfand-Fuks cohomology of the Lie algebra of compactly supported vectorfields on $R^{n}$ and establish the degeneracy of a certain spectral sequence at the $E_{1}$ level. We apply this result to the study of another spectral sequence introduced by Resetnikov for the cohomology of the algebra of vectorfields on $\boldsymbol{S}^{n}$.
\end{abstract}

Let $L$ be the Lie algebra of compactly supported smooth vectorfields on a manifold $M$. For $U$ a precompact open subset of $M$ let $L_{U}$ be the set of vectorfields supported in $U$ with the $C^{\infty}$ topology, then $L=U_{U \subset M} L_{U}$ and we give $L$ the topology of a strict inductive limit. Let $C^{q}(L)$ be the vectorspace of all continuous skewsymmetric R-multilinear functions from $L \times \cdots \times L$ ( $q$ times) into R. Define

$$
\begin{gathered}
d^{q}: C^{q}(L) \rightarrow C^{q+1}(L), \\
\left(d^{q} \lambda\right)\left(\xi_{1}, \ldots, \xi_{q+1}\right)=\sum(-1)^{i+j} \lambda\left(\left[\xi_{i}, \xi_{j}\right], \ldots, \hat{\xi}_{i}, \ldots, \hat{\xi}_{j}, \ldots, \xi_{q+1}\right)
\end{gathered}
$$

where $[$,$] denotes the Lie bracket of vectorfields and { }^{\wedge}$ indicates omission. Then $d^{q+1} \circ d^{q}=0$ and $C^{*}(L)=\bigoplus_{q=0, \ldots, \infty} C^{q}(L)$ is a differential complex with differential $d=\bigoplus d^{q}$. The cohomology of $\left(C^{*}(L), d\right)$ is known as the Gelfand-Fuks cohomology of $L$ with coefficients in $R$.

Let $\mathrm{pr}_{i}: M^{q} \rightarrow M$ be the projection on the $i$ th factor of the $q$-fold cartesian product of $M$ and let $\operatorname{pr}_{i}^{*} T$ be the pull-back of the tangent bundle to $M$ along $\mathrm{pr}_{i}$. Define $T^{q}=\mathrm{pr}_{1}^{*} T \otimes \cdots \otimes \mathrm{pr}_{q}^{*} T$ as a bundle over $M^{q}$. A vectorfield $\xi$ on $M$ defines a section $\operatorname{~pr}_{i}^{*} T$ in a natural way and a $q$-tuple $\left(\xi_{1}, \ldots, \xi_{q}\right)$ of vectorfields defines a section $\mathrm{pr}_{1}^{*} \xi_{1} \otimes \cdots \otimes \mathrm{pr}_{q}^{*} \xi_{q}$ of $T^{q}$ over $M^{q}$. Linear combinations of sections of this type are dense in the space of compactly supported sections of $T^{q}$, denoted $\left[T^{q}\right]_{C}$, with the inductive limit topology defined similarly to that on $L=[T]_{C}$. Thus an element $\lambda \in C^{q}(L)$ defines a continuous function

Received by the editors April 23, 1974 and, in revised form, December 10, 1974.

AMS (MOS) subject classifications (1970). Primary 18H25, 22E65, 58H05.

Key words and phrases. Continuous cohomology of a Lie algebra, Lie algebra of vectorfields, $\boldsymbol{k}$ jets of vectorfields, formal power series vectorfields, support of a distribution, degeneracy of a spectral sequence.

(1) Research partially supported by NSF grant GP-31917X1. 
$\tilde{\lambda}:\left[T^{q}\right]_{C} \rightarrow \mathbf{R}$. If $\operatorname{Hom}_{\mathbf{R}}\left(\left[T^{q}\right]_{C}, \mathbf{R}\right)$ denotes the continuous $\mathbf{R}$ multilinear functions, then we have a map $C^{q}(L) \rightarrow \operatorname{Hom}_{\mathbf{R}}\left(\left[T^{q}\right]_{C}, \mathbf{R}\right)$. If we let $B^{q}(L)$ denote the set of not necessarily skewsymmetric continuous $R$-multilinear functions $L \times \cdots \times L \rightarrow \mathbf{R}$, then we have an isomorphism:

$$
B^{q}(L) \cong \operatorname{Hom}_{\mathrm{R}}\left(\left[T^{q}\right]_{C}, \mathbf{R}\right) \text {. }
$$

Let $\Sigma_{q}$ be the permutation group on $q$-letters and corresponding to $\sigma \in \Sigma_{q}$ and $\lambda \in B^{q}(L)$ let $\sigma \circ \lambda \in B^{q}(L)$ be defined by

$$
(\sigma \circ \lambda)\left(\xi_{1}, \ldots, \xi_{q}\right)=\epsilon_{\sigma} \lambda\left(\xi_{\sigma(1)}, \ldots, \xi_{\sigma(q)}\right)
$$

where $\epsilon_{\sigma}$ is the sign of $\sigma$ as a permutation. With these definitions $C^{q}(L)$ is the subspace of $\Sigma_{q}$ invariants in $B^{q}(L)$.

$$
B^{q}(L)^{\Sigma}=C^{q}(L) \text {. }
$$

Let $D^{\prime}\left(M^{q}\right)$ be the space of distributions on $M^{q}$,

$$
D^{\prime}\left(M^{q}\right)=\operatorname{Hom}_{\mathrm{R}}\left(C_{0}^{\infty}\left(M^{q}\right), \mathbf{R}\right)=\operatorname{Hom}_{\mathrm{R}}\left([1]_{C}, \mathbf{R}\right) .
$$

Consider $C_{0}^{\infty}\left(M^{q}\right)$ as a left $C^{\infty}\left(M^{q}\right)$ module making $D^{\prime}\left(M^{q}\right)$ a right $C^{\infty}\left(M^{q}\right)$ module. Then

$$
\begin{aligned}
\operatorname{Hom}_{\mathrm{R}}\left(\left[T^{q}\right]_{C}, \mathbf{R}\right) & =\operatorname{Hom}_{\mathbf{R}}\left(\left[T^{q}\right] \otimes_{C^{\infty}\left(M^{q}\right)}[1]_{C}, \mathbf{R}\right) \\
& =\operatorname{Hom}_{C^{\infty}\left(M^{q}\right)}\left(\left[T^{q}\right], \operatorname{Hom}\left([1]_{C}, \mathbf{R}\right)\right) \\
& =\operatorname{Hom}_{C^{\infty}\left(M^{q}\right)}\left(\left[T^{q}\right], D^{\prime}\left(M^{q}\right)\right) \cong D^{\prime}\left(M^{q}\right) \otimes_{C^{\infty}\left(M^{q}\right)}\left[T^{q^{*}}\right] .
\end{aligned}
$$

Let $\Sigma_{q}$ act on $M^{q}$ by permuting factors $\sigma\left(x_{1}, \ldots, x_{q}\right)=\left(x_{\sigma^{-1}(1)}, \ldots, x_{\sigma^{-1}(q)}\right)$. This induces an action on $C_{0}^{\infty}\left(M^{q}\right)$ and by duality on $D^{\prime}\left(M^{q}\right)$. Let $\Sigma_{q}$ act on $T^{q^{*}}$ by permuting factors and multiplying by $\epsilon_{\sigma}$, then for $\omega_{1} \otimes \cdots \otimes \omega_{q} \in$ $\left[T^{q^{*}}\right], \xi_{1} \otimes \cdots \otimes \xi_{q} \in\left[T^{q}\right]_{C}$ and $u \in D^{\prime}\left(M^{q}\right)$,

$$
\begin{aligned}
\sigma(u \otimes & \left.\omega_{1} \otimes \cdots \otimes \omega_{q}\right)\left[\xi_{1} \otimes \cdots \otimes \xi_{q}\right] \\
& =\epsilon_{\sigma}\left(\sigma \circ u \otimes \omega_{\sigma^{-1}(1)} \otimes \cdots \otimes \omega_{\sigma^{-1}(q)}\right)\left[\xi_{1} \otimes \cdots \otimes \xi_{q}\right] \\
& =\epsilon_{\sigma}(\sigma \circ u)\left[\left\langle\omega_{\sigma^{-1}(1)}, \xi_{1}\right\rangle_{x_{1}} \circ \cdots \otimes\left\langle\omega_{\sigma^{-1}(q)}, \xi_{q}\right\rangle_{x_{q}}\right] \\
& =\epsilon_{\sigma} u\left[\left\langle\omega_{\sigma^{-1}(1)}, \xi_{1}\right\rangle_{x_{\sigma^{-1}}(1)} \cdots \cdots\left\langle\omega_{\sigma^{-1}(q)}, \xi_{q}\right\rangle_{x_{\sigma^{-1}(q)}}\right] \\
& =\epsilon_{\sigma} u\left[\left\langle\omega_{1}, \xi_{\sigma(1)}\right\rangle_{x_{1}} \circ \cdots \circ\left\langle\omega_{q}, \xi_{\sigma(q)}\right\rangle_{x_{q}}\right] \\
& =\epsilon_{\sigma} u \otimes \omega_{1} \otimes \cdots \otimes \omega_{q}\left[\xi_{\sigma(1)} \otimes \cdots \otimes \xi_{\sigma(q)}\right] .
\end{aligned}
$$

Therefore

$$
\left(D^{\prime}\left(M^{q}\right) \otimes_{C^{\infty}\left(M^{q}\right)}\left[T^{q^{*}}\right]\right)^{\Sigma} q \cong C^{q}(L)
$$


To compute the cohomology of $C^{q}(L)$ we use the spectral sequence defined as follows. Let $\left.D^{\prime}\left(M^{q}\right)\right|_{M^{q}}$ be the distributions with support on the subset $M_{k}^{q}=$ $\left\{\left(x_{1}, \ldots, x_{q}\right) \mid\right.$ at most $k$ of the points $\left.x_{1} \in M\right\}$. Set

$$
C_{k}^{q}(L)=\left(\left.D^{\prime}\left(M^{q}\right)\right|_{M_{k}^{q}} \otimes_{C^{\infty}\left(M^{q}\right)}\left[T^{q^{*}}\right]\right)^{\Sigma_{q}},
$$

then $C_{k}^{q}(L) \subset C_{k+1}^{q}(L)$ and $d^{q} C_{k}^{q}(L) \subset C_{k}^{q+1}(L)$. If we define $F^{-k} C^{q}=C_{k}^{q}$ we have a decreasing filtration preserved by the differential and thus a cohomology spectral sequence.

Note that $M_{k}^{q}$ is a union of submanifolds. In fact if $S$ is a partition of $q$ elements into $k$ sets, let $M_{S}^{q}$ be the set of points in $M^{q}$ consisting of $\left(x_{1}, \ldots, x_{q}\right)$ such that if $i, j$ are in the same subset of the partition then $x_{i}=x_{j}$. There is an obvious diffeomorphism of $M^{k}$ and $M_{S}^{q}$, and $M_{k}^{q}=\bigcup_{S}$ a partition of $k^{M_{S}^{q}}$. Any element of $\left.D^{\prime}\left(M^{q}\right)\right|_{M} ^{q}$ can be written as a sum of normal derivatives of distributions on $M_{S}^{q}$, see Schwartz [4]. P. Trauber in his Princeton thesis [6] has used the isomorphism (4) and this fact to give a nice description of the $E_{0}$ term of the spectral sequence and then applied the methods of relative homological algebra to compute $E_{1}$. We summarize his results below, making the obvious extension to the case of compactly supported vectorfields. Let $D(M)$ be the differential operators on $M$, not necessarily of finite order, topologized as follows. For $U$ a precompact open subset of $M$, let $D^{k}(U)$ be the differential operators of at most order $k$ on smooth functions with support in $U$. As sections of a vector bundle $D^{k}(U)$ has a nuclear locally convex topology and so the inductive limit $D(U)=$ $\lim _{k} D^{k}(U)$ does also. For $U \subset V$ there is a restriction map $D(V) \rightarrow D(U)$ and the precompact open subsets of $M$ together with these restriction maps form a directed system. Let $D(M)=\lim _{U C M} D(U)$, as a projective limit of nuclear spaces it is a nuclear space. If we use the cofinal family $U^{q}=U \times \cdots \times U$ ( $q$ times) of precompact open sets on $M^{q}$ to define the topology on $D\left(M^{q}\right)$, then because

$$
D^{k}\left(U^{q}\right) \cong D^{k}(U) \hat{\otimes} \cdots \hat{\otimes} D^{k}(U)
$$

and $\hat{\otimes}$ is an exact functor we have $D\left(U^{q}\right) \cong D(U) \hat{\otimes} \cdots \hat{\otimes} D(U)$ and $D\left(M^{q}\right) \cong$ $D(M) \hat{\otimes} \cdots \hat{\otimes} D(M)$. Similarly $\left[T^{q^{*}}\right] \cong\left[T^{*}\right] \hat{\otimes} \cdots \hat{\otimes}\left[T^{*}\right]$. Let $\left.D\left(M^{q}\right)\right|_{M_{S}^{q}}$ be the differential operators $C_{0}^{\infty}\left(M^{q}\right) \rightarrow C_{0}^{\infty}\left(M_{S}^{q}\right)$. Composition on the left defines a left $D\left(M_{S}^{q}\right)$ module structure on $\left.D\left(M^{q}\right)\right|_{M_{S}^{q}}$ and $C^{\infty}\left(M_{S}^{q}\right) \subset D\left(M_{S}^{q}\right)$. Relative to these structures we have the following

Proposition (Trauber [6]).

(a) $\left.\left.D^{\prime}\left(M^{q}\right)\right|_{M_{S}^{q}} \cong D^{\prime}\left(M_{S}^{q}\right) \otimes_{D\left(M_{S}^{q}\right)} D\left(M^{q}\right)\right|_{M_{S}^{q}}$,

(b) $\left.D\left(M^{q}\right)\right|_{M_{S}^{q}} \cong C^{\infty}\left(M_{S}^{q}\right) \otimes_{C^{\infty}\left(M^{q}\right)} D\left(M^{q}\right)$, where the $C^{\infty}\left(M^{q}\right)$ module structure on $C^{\infty}\left(M_{S}^{q}\right)$ is restriction followed by multiplication. Using these isomorphisms we have 


$$
\begin{aligned}
\left.D^{\prime}\left(M^{q}\right)\right|_{M_{S}^{q}} \otimes_{C^{\infty}\left(M^{q}\right)}\left[T^{\left.q^{*}\right]}\right. \\
\cong D^{\prime}\left(M_{S}^{q}\right) \otimes_{D\left(M_{S}^{q}\right)} C^{\infty}\left(M_{S}^{q}\right) \otimes_{C^{\infty}\left(M^{q}\right)} D\left(M^{q}\right) \otimes_{C^{\infty}\left(M^{q}\right)}\left[T^{\left.q^{*}\right]}\right. \\
\cong D^{\prime}\left(M_{S}^{q}\right) \otimes_{D\left(M_{S}^{q}\right)} C^{\infty}\left(M_{S}^{q}\right) \otimes_{C^{\infty}\left(M^{q}\right)}(D(M) \hat{\otimes} \cdots \otimes D(M)) \\
\otimes_{C^{\infty}(M) \hat{\otimes} \cdots \hat{\otimes} C^{\infty}(M)}\left(\left[T^{*}\right] \otimes \cdots \otimes\left[T^{*}\right]\right) \\
\cong D^{\prime}\left(M_{S}^{q}\right) \otimes_{D\left(M_{S}^{q}\right)} C^{\infty}\left(M_{S}^{q}\right) \otimes_{C^{\infty}\left(M^{q}\right)} D(M) \otimes_{C^{\infty}(M)}\left[T^{*}\right] \\
\quad \hat{\otimes} \cdots \hat{\otimes} D(M) \otimes_{C^{\infty}(M)}\left[T^{*}\right] .
\end{aligned}
$$

Let $D \otimes T^{*}=D(M) \otimes_{C^{\infty}(M)}\left[T^{*}\right]$ and let $X$ be the elements of positive degree in the exterior algebra over $C^{\infty}(M)$ of $D \otimes T^{*}$ let $X^{k}=X \hat{\otimes} \cdots \hat{\otimes} X$ ( $k$ times) and let $X^{k}(q)$ be the subspace of $X^{k}$ consisting of elements with $q$ factors of $T^{*}$. Trauber proves the following

THEOREM (Trauber [6]).

(a) $C_{k}^{q}(L) \cong\left(\left.D^{\prime}\left(M^{q}\right)\right|_{M_{k}^{q}} \otimes_{C^{\infty}\left(M^{q}\right)}\left[T^{\left.\left.q^{*}\right]\right)^{\Sigma} q} \cong\left(D^{\prime}\left(M^{k}\right) \otimes_{D\left(M^{k}\right)} X^{k}(q)\right)^{\Sigma_{k}}\right.\right.$,

$$
\frac{F^{-k} C^{*}(L)}{F^{-k+1} C^{*}(L)} \cong\left(\frac{D^{\prime}\left(M^{k}\right)}{\left.D^{\prime}\left(M^{k}\right)\right|_{M_{k-1}^{k}}} \otimes_{D\left(M^{k}\right)} X^{k}\right)^{\Sigma_{k}}
$$

He also points out the following interpretation of the isomorphism (a).

Let $J^{k}(T)$ be the bundle of $k$-jets on $M$, for $U$ a precompact open set let $\left[J^{k}(T)\right]_{U}$ be the sections with support in $U$, this is a Fréchet nuclear space. Define $\left[J^{\infty}(T)\right]_{C}=\lim _{U} \lim _{\leftarrow}\left[J^{k}(T)\right]_{U}$. This is a nuclear 1.c.s. such that

$$
D \otimes T^{*}=\operatorname{Hom}_{C^{\infty}(M)}\left(\left[J^{\infty}(T)\right]_{C}, C^{\infty}(M)\right) \text {. }
$$

There is a continuous function $j^{\infty}:[T]_{C} \rightarrow\left[J^{\infty}(T)\right]_{C}$ which associates to any compactly supported vectorfield its infinite jet at each point. The bundle $J^{\infty}(T)$ has a canonical connection $\nabla:\left[J^{\infty}(T)\right]_{C} \rightarrow\left[T^{*} \otimes J^{\infty}(T)\right]_{C}$ introduced by Spencer, see [2]. If $\tilde{\xi} \in\left[J^{\infty}(T)\right]_{C}$ then $\widetilde{\xi}=j^{\infty}(\xi)$ for some $\xi \in[T]_{C}$ if and only if $\nabla \tilde{\xi}=0$ in $\left[T^{*} \otimes J^{\infty}(T)\right]_{C}$. The connection $\nabla$ has 0 curvature and thus gives a representation of $D(M)$ on $\left[J^{\infty}(T)\right]_{C} \cdot\left({ }^{2}\right)$ The image of $j^{\infty}$ is the subspace of $D(M)$ invariants in $\left[J^{\infty}(T)\right]_{C}$. Using the isomorphism $D\left(M^{q}\right) \cong D(M) \hat{\otimes} \cdots$ $\hat{\otimes} D(M)$ we get a representation of $D\left(M^{q}\right)$ on $\left[J^{\infty}(T)\right]_{C} \hat{\otimes} \cdots \hat{\otimes}\left[J^{\infty}(T)\right]_{C}$,

(2) For any vector bundle $E$ with connection $\nabla: \underline{E} \rightarrow \underline{T^{*} \otimes E}$ we write $\nabla_{X}$ for the germ of a differential operator $\left(\nabla_{X} s\right)(p)=(\nabla S)(p)\left(X_{p}\right) \in \underline{E}_{p}$ where $X \in \underline{T}_{p}$ and $S \in E_{p}$. If $\nabla_{X} \nabla_{Y}-\nabla_{Y} \nabla_{X}-\nabla_{[X, Y]}=0$ we say the connection has curvature zero and we get a Lie algebra representation of $[T] \rightarrow[$ Diff $E]=$ differential operators on $E$. This extends to a representation $D(M) \rightarrow[$ Diff $E]$. 
which we will also denote by $\nabla$ also. For $\xi_{1} \otimes \cdots \otimes \xi_{q} \in\left[J^{\infty}(T)\right]_{C} \otimes \cdots \otimes$ $\left[J^{\infty}(T)\right]_{C}$ and $\eta_{1} \otimes \cdots \otimes \eta_{q} \in D(M) \otimes \cdots \otimes D(M)$,

$$
\nabla_{\eta_{1} \otimes \cdots \otimes \eta_{q}} \xi_{1} \otimes \cdots \otimes \xi_{q}=\nabla_{\eta_{1}} \xi_{1} \otimes \cdots \otimes \nabla_{\eta_{i}} \xi_{i} \otimes \cdots \otimes \nabla_{\eta_{q}} \xi_{q} .
$$

Now $L_{C} \stackrel{j \infty}{\longrightarrow}\left[J_{(\infty)}^{\infty}(T)\right]_{C}$ is a Lie algebra map; therefore there is a cocitain

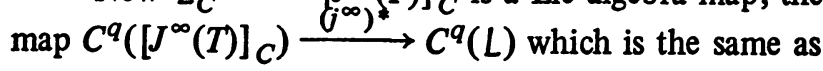

$D^{\prime}\left(M^{q}\right) \otimes_{C^{\infty}\left(M^{q}\right)}\left[J^{\infty}(T)\right]_{C}^{*} \hat{\otimes} \cdots \hat{\otimes}\left[J^{\infty}(T)\right]_{C}^{*} \stackrel{\left(j^{\infty}\right)^{*}}{\longrightarrow} D^{\prime}\left(M^{q}\right) \otimes_{C^{\infty}\left(M^{q}\right)}\left[T^{q^{*}}\right]$ or equivalently

$$
\begin{aligned}
D^{\prime}\left(M^{q}\right) \otimes_{C^{\infty}\left(M^{q}\right)} D \otimes T^{*} \hat{\otimes} \cdots \hat{\otimes} D \otimes T^{*} \\
\stackrel{\left(j^{\infty}\right)^{*}}{\longrightarrow} D^{\prime}\left(M^{q}\right) \otimes_{C^{\infty}\left(M^{q}\right)}\left[T^{q^{*}}\right] .
\end{aligned}
$$

Since the image of $j^{\infty}$ is the subspace of $D(M)$ invariants it is not hard to see that $\left(j^{\infty}\right)^{*}$ factors through the tensor product over $D\left(M^{q}\right)$ to give an isomorphism

$$
D^{\prime}\left(M^{q}\right) \otimes_{D\left(M^{q}\right)} D \otimes T^{*} \hat{\otimes} \cdots \hat{\otimes} D \otimes T^{*} \rightarrow D^{\prime}\left(M^{q}\right) \otimes_{C^{\infty}\left(M^{q}\right)}\left[T^{q^{*}}\right] .
$$

This allows us to identify the differential on the complex $X$ appearing in the previous theorem: $X$ is the exterior algebra on $\left[J^{\infty}(T)\right]_{C}^{*}$ and the differential $d_{X}$ on $X$ is the usual coboundary operator in the cochain complex on the dual of a Lie algebra. We can restate the previous theorem

$$
\begin{aligned}
\left(D^{\prime}\left(M^{k}\right) \otimes_{D\left(M^{k}\right)} \Lambda^{+}\left[J^{\infty}(T)\right]_{C}^{*} \hat{\otimes}\right. & \left.\cdots \hat{\otimes} \Lambda^{+}\left[J^{\infty}(T)\right]_{C}^{*}\right)^{\Sigma_{k}} \\
& \cong F^{-k} C^{*}(L) / F^{-k+1} C^{*}(L)
\end{aligned}
$$

as cochain complexes with the isomorphism induced by $\left(j^{\infty}\right)^{*}$.

To compute $H^{*}\left(F^{-k} / F^{-k+1}\right)$ we note that $X^{k}$ is flat as a $D\left(M^{k}\right)$ module since $X=\Lambda^{+} D \otimes T^{*}$ is flat as a $D$ module in each degree of the exterior power. Therefore the higher derived functors of $\otimes_{D\left(M^{k}\right)} X^{k}$ in the category of differential complexes vanish.

$$
\begin{aligned}
& \operatorname{Tor}_{p}^{D\left(M^{k}\right)}\left(A, X^{k}\right)=0, \quad p>0, \\
& \operatorname{Tor}_{0}^{D\left(M^{k}\right)}\left(A, X^{k}\right)=H^{*}\left(A \otimes_{D\left(M^{k}\right)} X^{k}, d_{X^{k}}\right) .
\end{aligned}
$$

However we can also compute the differential derived functor by resolving $X^{k}$. Let $Y_{p}=D\left(M^{k}\right) \otimes \Lambda^{p}\left[T\left(M^{k}\right)\right]$ define $\partial_{p}: Y_{p} \rightarrow Y_{p-1}$ by

$$
\begin{array}{r}
\partial_{p}\left(u \otimes \xi_{1} \wedge \cdots \wedge \xi_{p}\right)=\sum_{i}(-1)^{i-1} u \xi_{i} \otimes \xi_{1} \wedge \cdots \wedge \xi_{i} \wedge \cdots \wedge \xi_{p} \\
\cdot \sum_{i, j}(-1)^{1+j_{j}} \otimes \otimes\left[\xi_{i}, \xi_{j}\right] \wedge \xi_{1} \wedge \cdots \wedge \hat{\xi}_{i} \\
\wedge \cdots \wedge \xi_{j} \wedge \cdots \wedge \xi_{p} .
\end{array}
$$


Then $Y=\bigoplus Y_{p}$ gives a resolution of $C^{\infty}\left(M^{k}\right)$ as a left $D\left(M^{k}\right)$ module and tensoring on the right over $C^{\infty}\left(M^{k}\right)$ with $X^{k}$ we get a resolution:

$$
\left.D\left(M^{k}\right) \otimes_{C^{\infty}\left(M^{k}\right)}\right|_{\epsilon^{k}} ^{\Lambda\left[T\left(M^{k}\right)\right] \otimes_{C^{\infty}\left(M^{k}\right)} X^{k}}
$$

Let $A$ be a right $D\left(M^{k}\right)$ module then tensoring on the left over $D\left(M^{k}\right)$ with $A$

$$
\begin{gathered}
A \otimes_{C^{\infty}\left(M^{k}\right)} \Lambda^{*}\left[T\left(M^{k}\right)\right] \otimes_{C^{\infty}\left(M^{k}\right)} X^{k} \\
\quad \text { id } \otimes \epsilon_{0} \\
A \otimes_{D\left(M^{k}\right)} X^{k}
\end{gathered}
$$

as an augmented complex with homology (making $X^{k}$ a chain complex using negative indexing) equal to

$$
\operatorname{Tor}_{*}^{D\left(M^{k}\right)}\left(A, X^{k}\right)=H_{*}\left(A \otimes_{D\left(M^{k}\right)} X^{k}\right) .
$$

Computing the $\partial$ spectral sequence of the double complex we have

$$
E_{p,-q}^{1} \cong A \otimes \Lambda^{p}\left[T\left(M^{k}\right)\right] \otimes_{C^{\infty}\left(M^{k}\right)} H^{-q}\left(X^{k}\right) .
$$

Here we need an additional fact. Let $L$ be the algebra of formal power series vectorfields, i.e., the fiber of $J^{\infty}(T)$ over a point of $M, L=\lim _{\leftarrow} J^{k}(T)_{x}$. Let $L^{*}=\lim _{\rightarrow} J^{k}(T)_{x}^{*}$, then $H(X) \cong C^{\infty}(M) \otimes_{\mathrm{R}} H\left(\Lambda^{+} L^{*}\right)$ and the $\mathscr{D C}^{\leftarrow}(M)$ module structure on $H(X)$ is trivial, see [5] or [1a, pp. 205-206]. Therefore, we have

$$
H\left(X^{k}\right) \cong C^{\infty}\left(M^{k}\right) \otimes H\left(\Lambda^{+} L^{*} \otimes \cdots \otimes \Lambda^{+} L^{*}\right)
$$

with $D\left(M^{k}\right)$ acting trivially. Hence

$$
\begin{gathered}
E_{p,-q}^{2} \cong H\left(A \otimes_{C^{\infty}\left(M^{k}\right)} \Lambda\left[T\left(M^{k}\right)\right]\right) \otimes_{\mathrm{R}} H\left(\Lambda^{+} L^{*} \otimes \cdots \otimes \Lambda^{+} L^{*}\right), \\
E_{*}^{\infty} \cong \operatorname{Gr} H_{*}\left(A \otimes_{D\left(M^{k}\right)} X^{k}\right) .
\end{gathered}
$$

Let $\widetilde{D}^{\prime}\left(M^{k}-M_{k-1}^{k}\right)$ be the distributions on $M^{k}-M_{k-1}^{k}$ which extend to distributions on $M^{k}$. The inclusion $i: C_{0}^{\infty}\left(M^{k}-M_{k-1}^{k}\right) \rightarrow C_{0}^{\infty}\left(M^{k}\right)$ induces an isomorphism

$$
D^{\prime}\left(M^{k}\right) /\left.D^{\prime}\left(M^{k}\right)\right|_{M_{k-1}^{k}} \cong \widetilde{D}^{\prime}\left(M^{k}-M_{k-1}^{k}\right) \text {. }
$$

Since $\widetilde{D}^{\prime}\left(M^{k}-M_{k-1}^{k}\right)$ is dense in $D^{\prime}\left(M^{k}-M_{k-1}^{k}\right)$ and $D^{\prime}\left(M^{k}-M_{k+1}^{k}\right) \otimes_{C^{\infty}\left(M^{k}\right)}$ 
$\Lambda\left[T\left(M^{k}\right)\right]$ is dual to $\Omega_{C}\left(M^{k}-M_{k-1}^{k}\right)$ the de Rham complex of compactly supported differential forms we have a nondegenerate pairing

$$
\widetilde{D}^{\prime}\left(M^{k}-M_{k-1}^{k}\right) \otimes_{C^{\infty}\left(M^{k}\right)} \Lambda^{p}\left[T\left(M^{k}\right)\right] \times \Omega_{C}^{p}\left(M^{k}-M_{k-1}^{k}\right) \rightarrow \mathrm{R} .
$$

Moreover, the differential $\partial_{p}$ on the left factor is dual to the de Rham differential. Thus if $A=D^{\prime}\left(M^{k}\right) / D^{\prime}\left(M_{k-1}^{k}\right)$

$$
H_{p}\left(A \otimes_{C^{\infty}\left(M^{k}\right)} \Lambda\left[T\left(M^{k}\right)\right]\right) \cong H_{c}^{p}\left(M^{k}-M_{k-1}^{k}\right)^{*} .
$$

Putting all this together we conclude

THEOREM 1. Let $F^{-k} C^{*}(L) / F^{-k+1} C^{*}(L)$ be considered as a chain complex using negative indexing; then there is a homology spectral sequence with

$$
E_{p,-q}^{2} \cong\left(H_{C}^{p}\left(M^{k}-M_{k-1}^{k}\right) * \otimes H^{q}\left(\Lambda^{+} L^{*} \otimes \cdots \otimes \Lambda^{+} L^{*}\right)\right)^{\Sigma_{k}}
$$

and

$$
E_{p,-q}^{\infty}=\mathrm{Gr}_{p}\left(H^{q-p}\left(F^{-k} / F^{-k+1}\right)\right)
$$

In the special case when $M=\mathrm{R}^{n}$ we have $X \cong C^{\infty}(M) \otimes_{\mathrm{R}} \Lambda^{+} L^{*}$ and $X^{k} \cong$ $C^{\infty}\left(M^{k}\right) \otimes_{\mathrm{R}} \Lambda^{+} L^{*} \otimes \cdots \otimes \Lambda^{+} L^{*}$. This gives the following isomorphism

$$
\begin{aligned}
& D^{\prime}\left(M^{k}-M_{k-1}^{k}\right) \otimes_{C^{\infty}\left(M^{k}\right)} \Lambda\left[T\left(M^{k}\right)\right] \otimes_{C^{\infty}\left(M^{k}\right)} X^{k} \\
& \cong D^{\prime}\left(M^{k}-M_{k-1}^{k}\right) \otimes_{C^{\infty}\left(M^{k}\right)} \Lambda\left[T\left(M^{k}\right)\right] \otimes_{C^{\infty}\left(M^{k}\right)} C^{\infty}\left(M^{k}\right) \\
& \otimes_{\mathrm{R}} \Lambda^{+} L^{*} \otimes \cdots \otimes \Lambda^{+} L^{*} \\
& \cong\left(D^{\prime}\left(M^{k}-M_{k-1}^{k}\right) \otimes_{C^{\infty}\left(M^{k}\right)} \Lambda\left[T\left(M^{k}\right)\right]\right) \otimes_{\mathrm{R}}\left(\Lambda^{+} L^{*} \otimes \cdots \otimes \Lambda^{+} L^{*}\right)
\end{aligned}
$$

One can apply the Kunneth theorem to the latter complex, therefore its homology is

$$
H\left(D^{\prime}\left(M^{k}-M_{k-1}^{k}\right) \otimes \Lambda\left[T\left(M^{k}\right)\right]\right) \otimes_{\mathrm{R}} H^{*}\left(\Lambda^{+} L^{*} \otimes \cdots \otimes \Lambda^{+} L^{*}\right)
$$

and we conclude that $E^{2}=E^{\infty}$.

THEOREM 2. If $L$ is the Lie algebra of compactly supported vectorfields on $\mathbf{R}^{n}$, then with respect to the filtration defined earlier there is a spectral sequence with

$$
\begin{aligned}
E_{1}^{-k, l+k} & =H^{l} \frac{F^{-k} C^{*}(L)}{F^{-k+1} C^{*}(L)} \\
\cong & \bigoplus_{q-p=l}\left[H_{C}^{p}\left(\left(\mathrm{R}^{n}\right)^{k}-\left(\mathrm{R}^{n}\right)_{k-1}^{k}\right)^{*} \otimes_{\mathrm{R}} H^{q}\left(\Lambda^{+} L^{*} \otimes \cdots \otimes \Lambda^{+} L^{*}\right)\right]^{\Sigma_{k}}
\end{aligned}
$$


We will give an explicit expression for this isomorphism and show that the spectral sequence collapses at $E_{1}$.

When $M=\mathrm{R}^{n}$ we can find a global basis $\left[T\left(M^{k}\right)\right]$ as a $C^{\infty}\left(M^{k}\right)$ module which consists of commuting vectorfields; then

$$
\left[T\left(M^{k}\right)\right] \cong C^{\infty}\left(\mathrm{R}^{n k}\right) \otimes \mathrm{R}^{n k}, \quad \Lambda\left[T\left(M^{k}\right)\right] \cong C^{\infty}\left(\mathrm{R}^{n k}\right) \otimes_{\mathrm{R}} \Lambda \mathrm{R}^{n k}
$$

Let $\widetilde{X}^{k}=C^{\infty}\left(\mathrm{R}^{n k}\right) \otimes \Lambda(L \oplus \cdots \oplus L)^{*}$, i.e., the full exterior algebra. It is clear that $X^{k}$ is a direct summand of $\widetilde{X}^{k}$ as a $D\left(M^{k}\right)$ module. Let $j$ be the inclusion and $\pi$ the projection $X^{k} \stackrel{j}{\longrightarrow} \widetilde{X}^{k} \stackrel{\pi}{\longrightarrow} X^{k}$. Both $i$ and $\pi$ are cochain maps. Since $L \cong \mathbf{R}^{n} \oplus L^{0}$ we have $L \oplus \cdots \oplus L \cong \mathbf{R}^{n k} \otimes L^{0} \oplus \cdots \oplus L^{0}$ and there is an obvious interior product $\Lambda \mathrm{R}^{n k} \otimes_{\mathrm{R}} \widetilde{X}^{k} \rightarrow \widetilde{X}^{k}$. Using the isomorphisms given above we get a map

$$
\tilde{i} \Lambda\left[T\left(M^{k}\right)\right] \otimes_{C^{\infty}\left(M^{k}\right)} \tilde{X}^{k} \rightarrow \widetilde{X}^{k}
$$

Composing on the right with id $\otimes j$ and on the left with $\pi$ we get

$$
i: \Lambda\left[T\left(M^{k}\right)\right] \otimes_{C^{\infty}\left(M^{k}\right)} X^{k} \rightarrow X^{k}
$$

which we will denote

$$
\left.i: \xi_{1} \wedge \cdots \wedge \xi_{p} \otimes \alpha \mapsto \xi_{1} \wedge \cdots \wedge \xi_{p}\right\lrcorner \alpha .
$$

Tensoring on the left over $C^{\infty}\left(M^{k}\right)$ with $D\left(M^{k}\right)$

id $\otimes i: D\left(M^{k}\right) \otimes_{C^{\infty}\left(M^{k}\right)} \Lambda\left[T\left(M^{k}\right)\right] \otimes_{C^{\infty}\left(M^{k}\right)} X^{k} \rightarrow D\left(M^{k}\right) \otimes_{C^{\infty}\left(M^{k}\right)} X^{k}$. Composition with the left module structure on $X^{k}$ with $D\left(M^{k}\right) \otimes X^{k} \rightarrow X^{k}$ gives

$$
\begin{gathered}
\psi: D\left(M^{k}\right) \otimes_{C^{\infty}\left(M^{k}\right)} \Lambda\left[T\left(M^{k}\right)\right] \otimes_{C^{\infty}\left(M^{k}\right)} X^{k} \rightarrow X^{k} \\
\left.u \otimes \xi_{1} \wedge \cdots \wedge \xi_{p} \otimes \alpha \mapsto u\left(\xi_{1} \wedge \cdots \wedge \xi_{p}\right\lrcorner \alpha\right) .
\end{gathered}
$$

We will show that $\psi$ is a cochain map. Passing to $\Sigma_{k}$ invariants we get an explicit isomorphism for the $E^{1}$ term of the spectral sequence given in the previous theorem.

The map $\psi$ is defined with respect to a fixed parallelisation of $T\left(M^{k}\right)$, with respect to which we have

$$
\begin{aligned}
& D\left(M^{k}\right) \otimes_{C^{\infty}\left(M^{k}\right)} \Lambda\left[T\left(M^{k}\right)\right] \otimes_{C^{\infty}\left(M^{k}\right)} X_{k} \\
& \cong D\left(\mathrm{R}^{n k}\right) \otimes_{\mathrm{R}} \Lambda \mathrm{R}^{n k} \otimes_{\mathrm{R}} \Lambda^{+} L^{*} \otimes \cdots \otimes \Lambda^{+} L^{*} .
\end{aligned}
$$

The differential is given by

$$
\begin{aligned}
d\left(u \otimes \xi_{1} \wedge \cdots \wedge \xi_{p} \otimes \alpha\right)= & \sum(-1)^{i-1} u \xi_{i} \otimes \xi_{1} \wedge \cdots \wedge \hat{\xi}_{i} \wedge \cdots \wedge \xi_{p} \otimes \alpha \\
& +(-1)^{p} u \otimes \xi_{1} \wedge \cdots \wedge \xi_{p} \otimes d_{L} \alpha
\end{aligned}
$$


where $d_{L}$ is the differential in $\Lambda L^{*} \otimes \cdots \otimes \Lambda L^{*}$,

$$
\begin{aligned}
d \psi\left(u \otimes \xi_{1} \wedge\right. & \left.\cdots \wedge \xi_{p} \otimes \alpha\right) \\
= & \left.\left.d\left(u\left(\xi_{1} \wedge \cdots \wedge \xi_{p}\right\lrcorner \alpha\right)\right)=u d\left(\xi_{1} \wedge \cdots \wedge \xi_{p}\right\lrcorner \alpha\right) \\
= & u\left(\sum(-1)^{i-1}\left(\xi_{1} \wedge \cdots \wedge \hat{\xi}_{i} \wedge \cdots \wedge \xi_{p}\right\lrcorner \operatorname{ad} \xi_{i} \alpha\right) \\
& \left.\left.\quad+(-1)^{p}\left(\xi_{1} \wedge \cdots \wedge \xi_{p}\right\lrcorner d_{L} \alpha\right)\right) .
\end{aligned}
$$

By definition ad is the adjoint representation of $L \oplus \cdots \oplus L$ on $\Lambda(L \oplus \cdots \oplus L) *$ dual to the adjoint representation of $L \oplus \cdots \oplus L$ on $\Lambda(L \oplus \cdots \oplus L)$. For $\alpha \in \Lambda(L \oplus \cdots \oplus L)^{*}$ and $\xi_{1}, \ldots, \xi_{p} \in R^{n k}$ we have ad $\xi_{i} \alpha=\xi_{i} \cdot \alpha$ where indicates the module structure and $\xi_{i}$ are considered as constant coefficient differential operators. Furthermore $\left(\xi_{1} \wedge \cdots \wedge \xi_{i} \wedge \cdots \wedge \xi_{p}\right\lrcorner$ ad $\left.\xi_{i} \alpha\right)=$ ad $\left.\xi_{i}\left(\xi_{1} \wedge \cdots \wedge \hat{\xi}_{i} \wedge \cdots \wedge \xi_{p}\right\lrcorner \alpha\right)$, thus $\psi$ is a cochain map.

We can represent the induced map on cohomology

$\left[H_{c}^{p}\left(\mathrm{R}^{n k}-\left(\mathrm{R}^{n}\right)_{n-1}^{k}\right) \otimes_{\mathrm{R}} H^{q}\left(\Lambda^{+} L^{*} \otimes \cdots \otimes \Lambda^{+} L^{*}\right)\right]^{\Sigma_{k}} \rightarrow H^{q-p}\left(F^{-k} / F^{-k+1}\right)$

more conveniently as follows. For $\eta \in L, j^{\infty}(\eta) \in C_{0}^{\infty}(M) \otimes L$ so if $\alpha \in \Lambda L^{*}$ we can form $\left.j^{\infty}(\eta)\right\lrcorner \alpha \in C_{0}^{\infty}(M) \otimes \Lambda L^{*}$. For $\alpha=\Sigma \alpha_{1}^{i} \otimes \cdots \otimes \alpha_{k}^{i} \in \Lambda^{+} L^{*} \otimes$ $\cdots \otimes \Lambda^{+} L^{*}$ and for $S$ a partition $\left(a_{1}, \ldots, a_{s}\right)\left(a_{1}, \ldots, b_{s_{2}}\right) \cdots\left(c_{1}, \ldots, c_{s_{k}}\right)$ of $q$ into $k$ sets it makes sense to partition a set of $q$ vectorfield $\eta_{1}, \ldots, \eta_{q}$ into $\eta_{a_{1}}, \ldots, \eta_{a_{1}}, \ldots, \eta_{b_{1}}, \ldots, \eta_{b_{s 2}}, \ldots, \eta_{c_{1}}, \ldots, \eta_{c_{k k}}$ and form

$$
\begin{gathered}
\left.\left.\sum_{i}\left(j^{\infty}\left(\eta_{a_{1}}\right) \wedge \cdots \wedge j^{\infty}\left(\eta_{a_{s_{1}}}\right)\right\lrcorner \alpha_{1}^{i}\right) \wedge\left(j^{\infty}\left(\eta_{b_{1}}\right) \wedge \cdots \wedge j^{\infty}\left(\eta_{b_{s_{2}}}\right)\right\lrcorner \alpha_{2}^{i}\right) \\
\left.\wedge \cdots \wedge\left(j^{\infty}\left(\eta_{c_{1}}\right) \wedge \cdots \wedge j^{\infty}\left(\eta_{c_{s_{k}}}\right)\right\lrcorner \alpha_{k}^{i}\right) .
\end{gathered}
$$

We will write $\left.j^{\infty}\left(\eta_{1}\right) \wedge \cdots \wedge j^{\infty}\left(\eta_{q}\right)\right\lrcorner_{s} \alpha$ to mean the interior product just defined. Let $i: \mathbf{R}^{n k} \rightarrow L \oplus \cdots \oplus L$ be the injection defined earlier and $\Lambda(L \oplus \cdots \oplus L)^{*} \stackrel{i^{*}}{\longrightarrow} \Lambda \mathrm{R}^{n k^{*}}$ the extension of the dual map to exterior algebras. Let $\phi$ be the isomorphism

$$
C_{0}^{\infty}\left(\mathrm{R}^{n k}\right) \otimes \Lambda \mathrm{R}^{n k *} \stackrel{\phi}{\longrightarrow} \Omega_{c}\left(\mathrm{R}^{n k}\right)
$$

given by the choice of a parallelism. Finally for $S$, the partition above, let $\epsilon_{S}$ be the sign of the permutation

$$
\left(\begin{array}{l}
1 \cdots s_{1} \cdots k-s_{k}+1 \cdots k \\
a_{1} \cdots a_{s_{1}} \cdots c_{1} \cdots c_{s_{k}}
\end{array}\right)
$$

Then for $\lambda \in \widetilde{D}^{\prime}\left(\mathrm{R}^{n k}-\left(\mathrm{R}^{n}\right)_{k-1}^{k}\right) \otimes \Lambda^{p}\left[T\left(M^{k}\right)\right] \alpha \in\left(\Lambda^{+} L^{*} \otimes \cdots \otimes \Lambda^{+} L^{*}\right)^{q}$ we have 


$$
\begin{aligned}
\psi(\lambda \otimes \alpha)\left(\eta_{1}, \ldots, \eta_{q-p}\right) & \\
& \left.=\sum \epsilon_{S} \lambda\left[\phi i^{*}\left(j^{\infty}\left(\eta_{1}\right) \wedge \cdots \wedge j^{\infty}\left(\eta_{q-p}\right)\right\lrcorner_{S} \alpha\right)\right]
\end{aligned}
$$

and

$$
\psi(d \lambda \otimes \alpha)+(-1)^{q-p} \psi\left(\lambda \otimes d_{L} \alpha\right)=d_{0}(\psi(\lambda \otimes \alpha))
$$

where $d$ is the differential in $\widetilde{D}^{\prime}\left(M^{k}-M_{k-1}^{k}\right) \otimes \Lambda\left[T\left(M^{k}\right)\right], d_{L}$ is the differential in $\Lambda^{+} L^{*} \otimes \cdots \otimes \Lambda^{+} L^{*}$ and $d_{0}$ is the differential in $F^{k} C^{*}(L) / F^{-k+1} C^{*}(L)$.

Let $v_{i} \in \mathbf{R}^{n}$ and $\left(v_{1}, \ldots, v_{k}\right) \in \mathbf{R}^{n k}$ and let $\mathbf{R}_{(i, j)}^{n k-n}=\left\{\left(v_{1}, \ldots, v_{k}\right) \mid v_{i}\right.$ $\left.=v_{j}\right\}$ then $\left(\mathbf{R}^{n}\right)_{k-1}^{k}=\bigcup_{i<j} \mathbf{R}_{(i, j)}^{n k-n}$. Let $\mathbf{R}^{n k} \cup\{\infty\}=S^{n k}$ and $\mathbf{R}_{(i, j)}^{n k-n} \cup\{\infty\}=$ $S_{(i, j)}^{n k-n}$, then

$$
\begin{aligned}
H_{c}^{p}\left(\mathbf{R}^{n k}-\left(\mathrm{R}^{n}\right)_{k-1}^{k}\right) & =H_{c}^{p}\left(\mathbf{R}^{n k}-\bigcup_{i<j<k} \mathrm{R}_{(i, j)}^{n k-n}\right) \\
& =H_{c}^{p}\left(S^{n k}-\bigcup_{i<j<k} S_{(i, j)}^{n k-n}\right) \\
& \cong H^{p}\left(S^{n k}, \bigcup_{i<j<k} S_{(i, j)}^{n k-n}\right) .
\end{aligned}
$$

Hence

$$
H_{c}^{p}\left(\mathrm{R}^{n k}-\left(\mathrm{R}^{n}\right)_{k-1}^{k}\right)^{*} \cong H_{p}\left(S^{n k}, \bigcup_{i<j<k} S_{(i, j)}^{n k-n}\right)
$$

and composing these isomorphisms with $\psi$ we have

$$
\begin{array}{r}
\Phi:\left(H_{p}\left(S^{n k}, \bigcup_{i<j} S_{(i, j)}^{n k-n}\right) \otimes H^{q}\left(\Lambda^{+} L^{*} \otimes \cdots \otimes \Lambda^{+} L^{*}\right)\right)^{\Sigma_{k}} \\
\rightarrow E_{1}^{-k, q-p+k} .
\end{array}
$$

For $\Sigma_{l=1}^{m}\left[\sigma_{l}\right] \otimes\left[\alpha_{l}\right]$ an element of the left-hand side if we choose representative cycles $\sigma_{i}$ and representative cocycles $\alpha_{i}$ we get a representative element of $\Phi\left(\sum_{l=1}^{m}\left[\sigma_{l}\right] \otimes\left[\alpha_{l}\right]\right)$

$$
\begin{aligned}
& \left(\eta_{1}, \ldots, \eta_{q-p}\right) \\
& \left.\quad \mapsto \sum_{j=1}^{m} \int_{\sigma_{i}} \sum_{\text {partitions }} \epsilon_{S} \phi i^{*}\left(j^{\infty}\left(\eta_{1}\right) \wedge \cdots \wedge j^{\infty}\left(\eta_{q-p}\right)\right\lrcorner_{S} \alpha_{i}\right) .
\end{aligned}
$$

If we pull back $d_{1}: E^{-k, h+k} \rightarrow E^{-k+1, h+k}$ by the isomorphism $\Phi$ we get a mapping for $q-p=h$, 


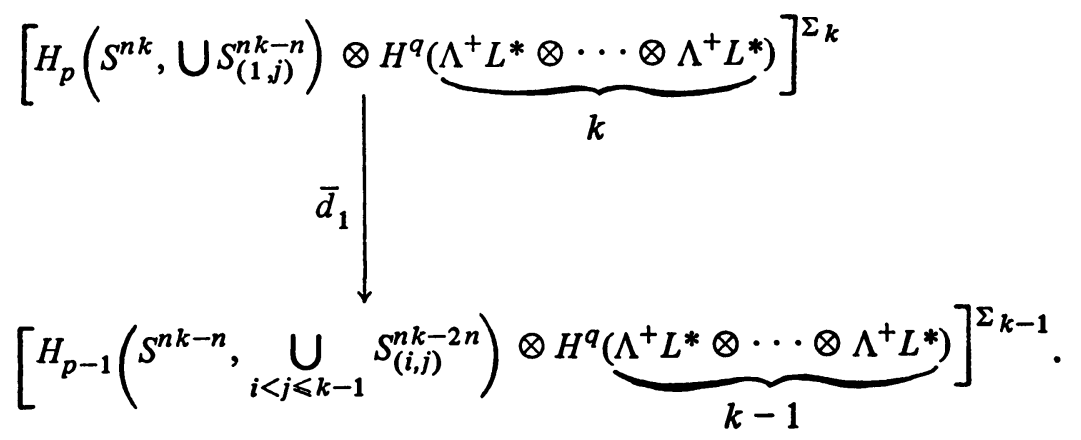

It is computed as follows. For $\eta_{1}, \eta_{2}, \ldots, \eta_{n+1} \in L$,

$$
\begin{aligned}
& \Phi\left(\bar{d}_{1} \sum_{l=1}^{m}\left[\sigma_{l}\right] \otimes\left[\alpha_{l}\right]\right)\left(\eta_{1}, \ldots, \eta_{h+1}\right) \\
& =\sum_{i<j \leqslant n+1}(-1)^{i+j} \Phi\left(\sum_{l=1}^{m}\left[\sigma_{l}\right] \otimes\left[\alpha_{l}\right]\right) \\
& \cdot \quad \cdot\left(\left[\eta_{i}, \eta_{j}\right], \eta_{1}, \ldots, \hat{\eta}_{i}, \ldots, \hat{\eta}_{j}, \ldots, \eta_{h+1}\right) \\
& =\sum_{i<j \leqslant n+1} \sum_{l} \sum_{S} \int_{\sigma_{l}} \epsilon_{S} \phi i^{*}\left(j^{\infty}\left(\left[\eta_{i}, \eta_{j}\right]\right) \wedge j^{\infty}\left(\eta_{1}\right) \wedge \cdots \wedge \widehat{j^{\infty}\left(\eta_{i}\right)}\right. \\
& \left.\left.\quad \wedge \cdots \wedge \hat{j}^{\infty}\left(\eta_{j}\right) \wedge \cdots \wedge j^{\infty}\left(\eta_{n+1}\right)\right\lrcorner_{S} \alpha_{l}\right) \\
& =\sum_{i<j \leqslant n+1} \sum_{l=1}^{m} \sum_{S} \int_{\sigma_{l}} \epsilon_{S} \phi i^{*}\left(\left[j^{\infty}\left(\eta_{i}\right), j^{\infty}\left(\eta_{j}\right)\right] \wedge j^{\infty}\left(\eta_{1}\right) \wedge \cdots \wedge \hat{j}^{\infty}\left(\eta_{i}\right)\right. \\
& \left.\left.\wedge \cdots \wedge \widehat{j^{\infty}\left(\eta_{j}\right)} \wedge \cdots \wedge j^{\infty}\left(\eta_{h+1}\right)\right\lrcorner_{S} \alpha_{l}\right) .
\end{aligned}
$$

Now $\alpha_{l}$ is a tensor product of $k$ cycles $\alpha_{l, j} \in Z\left(\Lambda^{+} L^{*}\right)$. To compute the last term we see what is happening to each $\alpha_{l, j}$. For $\alpha \in Z^{t}\left(\Lambda L^{*}\right)$ and $\eta_{1}, \ldots, \eta_{S}$ $\in L$

$$
\begin{aligned}
& \sum_{i<j \leqslant s}^{i+j} \phi i^{*}\left(\left[j^{\infty}\left(\eta_{i}\right), j^{\infty}\left(\eta_{j}\right)\right] \wedge j^{\infty}\left(\eta_{1}\right) \wedge \cdots \wedge \widehat{j^{\infty}\left(\eta_{i}\right)}\right. \\
& \left.\left.\wedge \cdots \wedge \widehat{j\left(\eta_{j}\right)} \wedge \cdots \wedge j^{\infty}\left(\eta_{s+1}\right)\right\lrcorner \alpha\right) \\
& =\sum_{i<j \leqslant s} \sum_{i_{1}<i_{2}<\cdots<i_{t-s} \leqslant n}(-1)^{i+j} \alpha\left(\left[j^{\infty}\left(\eta_{i}\right), j^{\infty}\left(\eta_{j}\right)\right], j^{\infty}\left(\eta_{1}\right) \cdots \widehat{j^{\infty}\left(\eta_{i}\right)}\right. \\
& \left.\cdots \widehat{j^{\infty}\left(\eta_{j}\right)} \cdots j^{\infty}\left(\eta_{s}\right), e_{i_{1}} \cdots e_{i_{t-s}}\right) \\
& d x^{i_{1}} \wedge \cdots \wedge d x^{i_{t-s}}
\end{aligned}
$$




$$
\begin{gathered}
\text { COMPACTLY SUPPORTED VECTORFIELDS ON R } \\
+\sum_{r, j} \sum_{i_{1}<i_{2}<\cdots<i_{t-s}<n}(-1)^{r+s+j} \alpha\left(\left[e_{i_{r}}, j^{\infty}\left(\eta_{j}\right)\right], j^{\infty}\left(\eta_{1}\right) \cdots \hat{j}^{\infty}\left(\eta_{j}\right)\right. \\
\left.\cdots j^{\infty}\left(\eta_{s}\right), e_{i_{1}} \cdots \hat{e}_{i_{r}} \cdots e_{i_{t-s}}\right) \\
d x^{i_{1}} \wedge \cdots \wedge d x^{i_{t-S}} \\
+\sum_{r, j} \sum_{i_{1}<i_{2}<\cdots<i_{t-S^{<n}}} \frac{\partial}{\partial x^{i_{r}}} \alpha\left(j^{\infty}\left(\eta_{1}\right), \ldots, j^{\infty}\left(\eta_{S}\right), e_{i_{1}} \cdots \hat{e}_{i_{r}} \cdots e_{i_{t-S}}\right) \\
\left.=d \phi i^{*}\left(j^{\infty}\left(\eta_{1}\right) \wedge \cdots \wedge j^{\infty}\left(\eta_{S}\right)\right\lrcorner \alpha\right) .
\end{gathered}
$$

This shows what happens to each factor of $\alpha_{l}$; hence the end product is

$$
\begin{aligned}
\Phi\left(\bar{d}_{1} \sum\left[\sigma_{l}\right] \otimes\left[\alpha_{l}\right]\right)\left(\eta_{1}, \ldots, \eta_{h+1}\right) \\
\left.=\sum_{i} \cdot \sum_{S^{\prime}} \int_{\sigma_{l}} \epsilon_{S^{\prime}} d \phi i^{*}\left(j^{\infty}\left(\eta_{1}\right) \wedge \cdots \wedge j^{\infty}\left(\eta_{n+1}\right)\right\lrcorner_{S^{\prime}} \alpha\right) \\
\left.=\sum_{l} \sum_{S^{\prime}} \int_{\partial \sigma_{l}} \epsilon_{S^{\prime}} \phi i^{*}\left(j^{\infty}\left(\eta_{1}\right) \wedge \cdots \wedge j^{\infty}\left(\eta_{n+1}\right)\right\lrcorner_{S^{\prime}} \alpha\right)
\end{aligned}
$$

where $S^{\prime}$ ranges over partitions of $h+1$ elements into $k$ sets. We can decompose $\partial \sigma_{l}$ into a sum of $\partial_{(i, j)} \sigma_{l}$ where $\left|\partial_{(i, j)} \sigma_{l}\right| \subset S_{(i, j)}^{n k-n}$. When $\phi i^{*}\left(j^{\infty}\left(\eta_{1}\right) \wedge \cdots \wedge\right.$ $\left.\left.j^{\infty}\left(n_{n+1}\right)\right\lrcorner_{S} \alpha\right)$ is integrated over $S_{(i, j)}^{n k-n}$, the $i$ th and $j$ th factors are identified by restricting to the diagonal in the product of the $i$ th and $j$ th factors. This gives a mapping

$$
\begin{aligned}
& H(\underbrace{\Lambda^{+} L^{*} \otimes \cdots \otimes \Lambda^{+} L^{*}}_{k}) \\
& \qquad \underbrace{}_{k-1} \cong H(\underbrace{\Lambda^{+} L^{*} \otimes \cdots \otimes \Lambda^{+} L^{*}}_{k}) \cong H(\underbrace{\left.\Lambda^{+} L^{*}\right) \otimes \cdots \otimes H\left(\Lambda^{+} L^{*}\right.}_{k-1})
\end{aligned}
$$

by multiplying the $i$ th and $j$ th factors, just as restriction to the diagonal induces the cup product in singular cohomology. Therefore the $\bar{d}_{1}$ operator involves multiplication in the cohomology algebra of the formal Lie algebra. It is known that this multiplication is trivial [5], [7], so $\bar{d}_{1}=0$. In a similar way one can see that all the higher differentials involve multiplication in the formal algebra so we have

THEOREM 3. There is a spectral sequence for the continuous cohomology of the algebra of compactly supported vectorfields on $\mathbf{R}^{n}$ which collapses at the $E_{1}$ level.

$$
E^{-k, l+k} \cong\left[\bigoplus_{q-p=l} H_{p}\left(S^{n k}, \bigcup_{i<j} S_{i, j}^{n k-n}\right) \otimes \bigotimes^{k} H^{+}(L)\right]^{\Sigma_{k}}
$$


Let $L$ be the algebra of vectorfields on the $n$ sphere $S^{n}$, let $p \in S^{n}$ and let $\tilde{L}$ be the ideal of vectorfields flat at $p$ in some, hence any, coordinate system. Let $C^{*}(L)$ be the Gelfand-Fuks complex for the continuous cohomology of $L$, and define a filtration

$$
F^{k} C^{q}(L)=\left\{\lambda \in C^{q}(L) \mid \lambda\left(\xi_{1}, \ldots, \xi_{q}\right)=0 \text { if } q-k+1 \text { of } \xi_{i} \text { are in } \tilde{L}\right\},
$$

then $F^{k} \supset F^{k+1}$ and $d F^{k} \subset F^{k}$. This is the filtration defining the HochschildSerre spectral sequence for $H(L)$ with respect to the ideal $\tilde{L}$.

$$
E_{2}^{p, q} \cong H^{p}\left(L / \tilde{L}, H^{q}(\tilde{L})\right), \quad E_{\infty}^{p, q} \simeq \mathrm{Gr}_{p}\left(H^{p+q}(L)\right)
$$

There is an exact sequence of Lie algebras

$$
0 \rightarrow \tilde{L} \rightarrow L \rightarrow L \rightarrow 0 \text {. }
$$

Thus $E_{2}^{p, q} \cong H^{p}\left(L, H^{q}(\tilde{L})\right)$. The action of $L$ on $H^{q}(\tilde{L})$ is defined as follows: for $\eta \in L$ let $\bar{\eta} \in L$ be a vectorfield such that $j^{\infty}(\bar{\eta})_{p}=\eta$ then Lie derivation with respect to $\bar{\eta}$ defines a map $D_{\bar{\eta}}: \widetilde{L} \rightarrow \widetilde{L}$ which in turn defines a cochain map $D_{\bar{\eta}}: C^{*}(\tilde{L}) \rightarrow C^{*}(\tilde{L})$ and therefore a map $D_{\bar{\eta}}^{*}: H^{*}(\widetilde{L}) \rightarrow H^{*}(\tilde{L})$. If $j^{\infty}\left(\bar{\eta}_{1}\right)_{p}=j^{\infty}\left(\bar{\eta}_{2}\right)_{p}$ then $\bar{\eta}_{1}-\bar{\eta}_{2} \in \tilde{L}$ and as is well known $D_{\bar{\eta}_{1}-\bar{\eta}_{2}}$ induces the trivial map in cohomology, so $D_{\bar{\eta}_{1}}^{*}=D_{\bar{\eta}_{2}}^{*}$. Resetnikov [3] has stated the following theorem for arbitrary $M$ but it is not clear to us that his proof is correct.

THEOREM. Since $L$ acts trivially on $H^{*}(\tilde{L})$, the $E_{2}$ term of the previous spectral sequence is $E_{2}^{p, q} \cong H^{p}(L) \otimes H^{q}(\widetilde{L})$. Furthermore if $L_{C}$ is the algebra of compactly supported vectorfields on $\mathbf{R}^{n}$, then $H^{q}(\tilde{L}) \cong H^{q}\left(L_{C}\right)$.

Proof. Let $\left\{U_{i}\right\}$ be a decreasing sequence of open sets which form a neighborhood basis at $p$. Let $K_{i}=S^{n}-U_{i}$; then $K$ is compact, and if we define $\phi: S^{n}-\{p\} \rightarrow R^{n}$ by stereographic projection with $p$ as north pole then the $\phi\left(K_{i}\right)$ form a compact exhaustion of $\mathbf{R}^{n}$. Let $L_{i}$ be the algebra of vectorfields on $S^{n}$ with support in $K_{i}$, there are inclusions $\tau_{j}^{i}: L_{i} \rightarrow L_{j}$; therefore, we can define $L_{\infty}=\lim _{\rightarrow} L_{i}$. Clearly $L_{\infty} \cong L_{C}$, compactly supported vectorfields. Let $\psi^{i}: L_{i}$ $\rightarrow \tilde{L}$ be the inclusion; then $\psi^{j} \cdot \tau_{j}^{i}=\psi^{i}$ so we can define $\psi: L_{\infty} \rightarrow \tilde{L}$. This induces $\psi^{*}: H(\widetilde{L}) \rightarrow H\left(L_{\infty}\right)$. For $\eta \in L$ let $\bar{\eta}_{i} \in L$ be a vectorfield such that $j^{\infty}\left(\bar{\eta}_{i}\right)_{p}=\eta$ and supp $\bar{\eta}_{i} \subset U_{i}$; then for $\lambda \in H^{*}(\tilde{L})$ we have $\eta \cdot[\lambda)=\left[D_{\bar{\eta}_{i}}^{*} \lambda\right]$ for any $i$. Clearly $\psi^{i *}\left[D_{\eta_{i}}^{*} \lambda\right]=0$ and from the fact that $\psi^{*} \eta \cdot[\lambda]=0$ if and only if $\left(\psi^{i}\right)^{*} \eta \cdot[\lambda]=0$ for all $i$ we conclude $\psi^{*} \eta \cdot[\lambda]=0$. To conclude the proof it is sufficient to show that $\psi^{*}$ is injective. In fact, $\psi^{*}$ is an isomorphism. To see this, look at the spectral sequences defined at the beginning of the paper. Since $\tilde{L}$ can be thought of as rapidly decreasing vectorfields on $\mathbf{R}^{n}$, the space that arises in defining $C^{*}(\widetilde{L})$ is $S^{\prime}\left(R^{n}\right)$. From this observation we see that the spectral sequence converging to $H^{*}\left(F^{-k} C^{*}(\widetilde{L}) / F^{-k+1} C^{*}(\tilde{L})\right)$, which is $E_{1}$ of 
another spectral sequence, has $E_{p,-q}^{2}$,

$$
\left[H_{p}\left(S^{\prime}\left(\mathbf{R}^{n k} /\left.S^{\prime}\left(\mathbf{R}^{n k}\right)\right|_{\left(\mathbf{R}^{n}\right)_{k-1}^{k}}\right) \otimes \Lambda\left[T\left(\mathbf{R}^{n k}\right)\right]\right) \otimes H^{q}\left(\Lambda^{+} L^{*} \otimes \cdots \otimes \Lambda^{+} L^{*}\right)\right]^{\Sigma_{k}} \text {. }
$$

We can identify the factor on the left from the following exact sequences (see Schwartz [4]). If $p$ is the north pole of $S^{n k}$,

$$
\begin{aligned}
& \left.0 \rightarrow E^{\prime}\left(S^{n k}\right)\right|_{p} \rightarrow E^{\prime}\left(S^{n k}\right) \rightarrow S^{\prime}\left(\mathrm{R}^{n k}\right) \rightarrow 0 \\
& \left.0 \rightarrow E^{\prime}\left(S^{n k}\right)\right|_{p} \rightarrow E^{\prime}\left(S^{n k}\right)\left|\underset{i<j \leqslant k}{\bigcup_{(i, j)} S^{n k-n}} S^{\prime}\left(\mathrm{R}^{n k}\right)\right| \underset{\left(\mathrm{R}^{n}\right)_{k-1}^{k}}{\longrightarrow} 0 .
\end{aligned}
$$

Thus

$$
H_{p}\left(S^{\prime}\left(\mathrm{R}^{n k} /\left.S^{\prime}\left(\mathrm{R}^{n k}\right)\right|_{\left.\left(\mathrm{R}^{n}\right)_{k-1}^{k}\right)} \otimes \Lambda\left[T\left(\mathrm{R}^{n k}\right)\right]\right) \cong H_{p}\left(S^{n k}, U S_{(i, j)}^{k-n}\right)\right.
$$

and $E_{p,-q}^{2}(\tilde{L}) \cong E_{p,-q}^{2}\left(L_{\infty}\right)$.

\section{BIBLIOGRAPHY}

1a. I. M. Gel'fand and D. B. Fuks, Cohomologies of the Lie algebra of tangent vector fields of a smooth manifold. I, Funkcional. Anal. i Priložen. 3 (1969), no. 3, 32-52 = Functional Anal. Appl. 3 (1969), 194-210. MR 41 \#1067.

1b. - Cohomologies of the Lie algebra of tangent vector fields. II, Funkcional. Anal. i Priložen. 4 (1970), no. 2, 23-31 = Functional Anal. Appl. 4 (1970), 110-116. MR 44 \#2248.

2. A. Kumpera and D. C. Spencer, Lie equations, Ann. of Math. Studies, Princeton Univ. Press, Princeton, N. J., 1973.

3. V. N. Rešetnikov, On the conomology of the Lie algebra of vector fields, Dokl. Akad. Nauk SSSR 208 (1973), 1041-1043 = Soviet Math. Dokl. 14 (1973), 234-240. MR 48 \#1231.

4. L. Schwartz, Théorie des distributions, Hermann, Paris, 1966. MR 35 \#30.

5. S. Shnider, Notes on Gel'fand-Fuks cohomology, Available from Math. Dept, Princeton University, Princeton, N. J.

6. P. Trauber, Continuous cohomology of the Lie algebra of vector fields, Thesis, Princeton University, Princeton, N. J., 1973.

7. C. Godbillon, Cohomologie d'algèbres de Lie de champs de vecteurs formels, Séminaire Bourbaki 1972/73, Exposé 421.

DEPARTMENT OF MATHEMATICS, PRINCETON UNIVERSITY, PRINCETON, NEW JERSEY 08540 